Cite this: J. Mater. Chem. B, 2021, 9, 392

Received 22nd September 2020, Accepted 29th October 2020

DOI: $10.1039 / d 0 t b 02279 a$

rsc.li/materials-b
Check for updates

\section{Design of biodegradable bi-compartmental microneedles for the stabilization and the controlled release of the labile molecule collagenase for skin healthcare $\dagger$}

\author{
Concetta Di Natale, $\ddagger^{\mathrm{ab}}$ Domenico De Rosa, $\ddagger^{\mathrm{a}}$ Martina Profeta, ${ }^{\mathrm{a}}$ Rezvan Jamaledin, ${ }^{\mathrm{a}}$ \\ Alessandro Attanasio, ${ }^{a}$ Elena Lagreca, ${ }^{a}$ Pasqualina Liana Scognamiglio, ${ }^{a}$ \\ Paolo Antonio Netti ${ }^{\text {ab }}$ and Raffaele Vecchione (iD *a
}

\begin{abstract}
Proteins are widely explored as therapeutic agents, but some issues remain alive in their delivery versus target tissues and organs. Especially in the case of water-labile proteins, they undergo rapid failure if not properly stored or once they have encountered the biological environment. In this framework, delivery systems can be very useful to protect such proteins both during storage and during their administration. In particular, polymer microneedles (MNs) represent an interesting tool for the in vivo administration of proteins, avoiding the aggressive gastrointestinal or blood environment. Here, polymer microneedles for the encapsulation and delivery of the labile protein collagenase are presented. Polyvinylpyrrolidonehyaluronic acid (PVP-HA) microneedles with embedded poly(lactic-co-glycolic acid) (PLGA) microparticles (MPs) were designed in order to achieve a sustained but relatively fast release of the enzyme to avoid its long exposure to water upon administration. PLGA-MPs with tunable porosity were produced by means of a modified double emulsion protocol and their morphological and kinetic properties were characterized by different analytic techniques. Diffusion studies and in vivo experiments were used to assess the release and indentation ability of the proposed MP-based microneedles. The obtained results recommend our bi-compartmental system as a promising biomedical technique paving the way for its efficient use in treating human diseases with labile therapeutic agents.
\end{abstract}

\section{Introduction}

During the last decade, biotechnology has established a pivotal role in pharmaceutical research for drug development, bringing protein-based products onto the market at an increasing pace. ${ }^{1}$ Molecular biology together with new chemical engineering techniques give us novel tools to increase the range of protein-based drugs to treat several diseases. ${ }^{2,3}$ Unfortunately, these products suffer from many disadvantages, including complex metabolic properties and restricted gastrointestinal (GI) absorption. In addition, they show difficult tissue penetration (because of their molecular weight and conformation), and toxicity due to the stimulation of the immune system that leads

\footnotetext{
${ }^{a}$ Center for Advanced Biomaterials for HealthCare@CRIB, Istituto Italiano di Tecnologia, Largo Barsanti e Matteucci 53, Napoli 80125, Italy. E-mail: Raffaele.vecchione@iit.it

${ }^{b}$ Interdisciplinary Research Center of Biomaterials, CRIB, University Federico II, P.le Tecchio 80, Naples 80125, Italy

$\dagger$ Electronic supplementary information (ESI) available. See DOI: 10.1039/ d0tb02279a

\# These authors contributed equally.
}

to allergic reactions. ${ }^{4}$ Moreover, proteins used as drugs often possess a short plasma half-life and, above all, they are highly susceptible to degradation in both physical and biological environments. $^{5-7}$ These alterations involve the primary structure of the proteins leading to a conformational change which results in reduction in protein activity. As protein drugs continue to play a key role in pharmaceuticals, their long-term stability is an urgent challenge for researchers. In this context, microparticle-based formulations can be developed to improve the storage and delivery of protein-drugs. Microparticle-based systems include a biodegradable polymeric material that allows both drug cargo protection and its controlled release. ${ }^{8}$ Several polymers can be used as a matrix, but poly(lactic-co-glycolic) acid (PLGA) is the most commonly studied polymer due to its versatility in tuning biodegradation time and high biocompatibility arising from its bio-units: lactic acid and glycolic acid. ${ }^{8,9}$ Protein-embedded polymeric microparticles (MPs) have already demonstrated to be able to effectively protect the encapsulated protein from inactivation occurring in biological environments and to preserve its bioactivity during the release process. ${ }^{10}$ Release can be easily tuned by modifying the size and the microstructure 
of MPs by experimental or mathematical protocols. ${ }^{11-13}$ The preservation of protein bioactivity reduces the administration frequency, leading to patient comfort and improvements in compliance, which are fundamental for a successful selfadministered drug delivery system. ${ }^{14}$ To overcome the known setbacks associated with oral as well as injectable formulations, transdermal delivery certainly offers a potential alternative. However, MPs with a diameter greater than $1 \mu \mathrm{m}$ barely penetrate into the lipid layers of the stratum corneum, thus limiting the drug transport through the skin. ${ }^{15}$ Several techniques have been investigated to assist the delivery of MPs through the skin. Among these, successful transdermal patches formed by micron-scale needles have been developed and proposed so far. ${ }^{16}$ Several drug delivery studies have emphasized the use of microneedles (MNs), thanks to their capability to increase skin permeability both in vitro and in vivo. Delivery of oligonucleotides, lipophilic molecules, diabetes treatment, and the induction of immune responses from protein vaccines are only some of the results achieved by using them. ${ }^{17}$ The mechanism of MNs is to pierce holes into the skin, avoiding the stimulation of the nerve endings in order to quickly absorb the biomolecules in the lymphatic networks without producing patient pain. ${ }^{18,19}$ Very recently, a novel fast stamp-based method for fabricating multi-compartmental polymeric microneedles has been developed in our laboratory. In detail, the body of the MN was filled with PLGA-based MPs thus combining the fast release of a molecule loaded in the tip with a time-controlled release of the payload from the MPs as well as the potential co-delivery of different biomolecules. ${ }^{20}$

Normally the release of biomolecules from PLGA drug delivery systems takes from several hours up to a few days, but in the case of highly labile molecules, such as collagenase, it is important that the sustained release occurs within a few hours. ${ }^{21}$ Moreover, in several pathologies, such as wound healing, burns, and some diseases including Dupuytren's disease, intervertebral disc herniation, keloids, cellulite, and lipoma, collagen can be generated in unsuitable sites or does not degrade after a certain time, ${ }^{22}$ so it is important to have a device capable of injecting collagenase into the required site or at the right time.

Here, we present a system of bi-compartmental microneedles in which a novel formulation of highly porous collagenaseembedded PLGA MPs has been inserted in order to guarantee prolonged release, but in a timeframe of a few hours, of the enzyme that usually suffers from high instability in an aqueous environment. ${ }^{22}$ Unlike the previously developed protocol, ${ }^{19}$ the polyvinylpyrrolidone (PVP) tip that is sensitive to the varying humidity and storage time ${ }^{23}$ has been replaced with a less fragile PVP-hyaluronic acid (PVP-HA) mixture to allow strong dehydration which is useful for preserving the protein from humidity. A second main change regarding PLGA MPs is that they were synthesized by a modified double emulsion protocol $^{13}$ to tune their porosity, and thus speed up cargo release. The morphological properties of the MPs were characterized by a Mastersizer and scanning electron microscopy (SEM). Moreover, collagenase encapsulation efficiency, kinetic release, and its retained activity were investigated by spectroscopic techniques such as fluorescence and UV-visible. Furthermore, through in vitro diffusion studies we evaluated the release ability of the two microneedle compartments (tip and MP-based body) within the established times of a few hours. A preliminary in vivo test confirmed the ability of the microneedles to by-pass the stratum corneum of rat epidermis.

These results suggest that microneedles combined with MPs represent a promising technology for the healthcare field, especially for skin pathologies. It has indeed been proven that some skin pathologies, such as skin burns, atopic dermatitis or infantile eczema, cause a rapid loss of plasma proteins and their intravenous transfusion is the most effective therapy. ${ }^{24,25}$ Unfortunately, since plasma proteins are labile molecules, they are often degraded before carrying out their action, greatly reducing their therapeutic power. ${ }^{26}$ Our platform can be placed in this scenario as a good alternative to deliver labile molecules into the skin, protecting them from degradation and therefore improving their healing effects.

\section{Results and discussions}

Polymer-based biomaterials for the delivery of water-labile proteins have attracted significant interest in the biomedical field. The fabrication processes to obtain the final drug delivery system should be flexible and compatible with the stability of the protein of interest. In the following paragraphs, we will explain a novel bottom-up approach in which we first encapsulated and characterized from a structural and functional point of view collagenase protein into PLGA-based MPs obtained by a double emulsion technique. Later, we fabricated bi-compartmental microneedles embedding such MPs for intradermal drug delivery. Furthermore, the collagenase biodistribution, released from MPs embedded into the body of the microneedles, has been studied through both in vitro and in vivo analysis, showing the potential of the proposed microneedle-based tool for the delivery of labile molecules.

\subsection{Collagenase functionalization with ATTO 488 and ATTO 740: reaction, CD spectroscopy, and activity assay}

Biomolecule fluorophore conjugation involving lysine residues is a powerful tool for biochemical, biophysical and analytical assays. $^{27}$ Unfortunately, some proteins, e.g. collagenase, are sensitive to buffers, metal ions, or $\mathrm{pH}$ exchanges, incurring aggregation or precipitation phenomena. ${ }^{28} \mathrm{~A}$ common approach to overcome these limitations is to label solventaccessible cysteine residues using thiol-reactive dyes. Cysteine is very attractive for site-specific conjugation because of its relative rarity in the proteome compared to amines; this feature leads to less perturbation of the protein's function and a minor risk of incurring protein precipitation or fluorescence self-quenching interactions. ${ }^{29}$ Starting from these proofs, a maleimide-based conjugation of collagenase with two different fluorophores: ATTO 488 (for in vitro analysis) and ATTO 740 (for in vivo analysis) was reported for the first time following the 
A

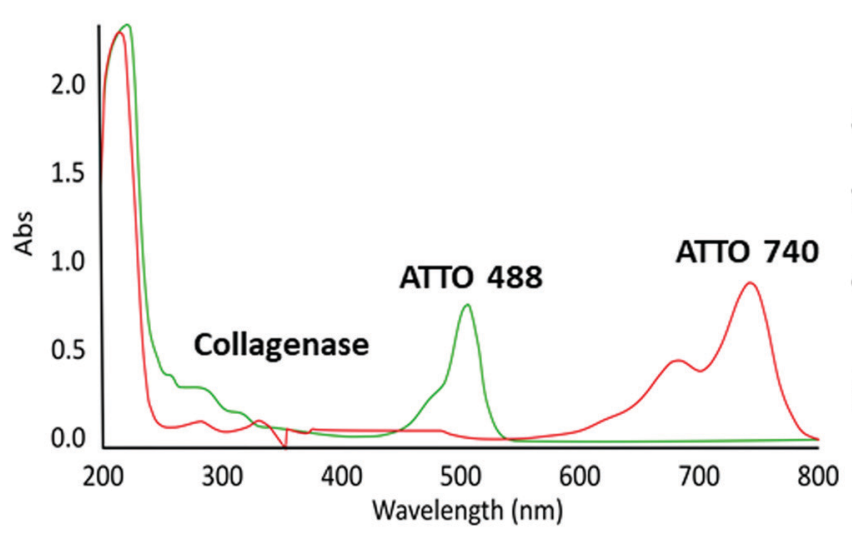

B

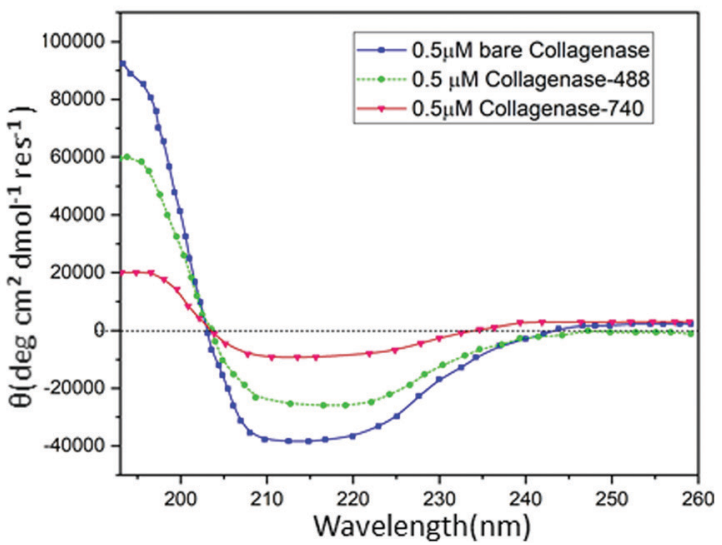

Fig. 1 (A) UV spectra of collagenase-ATTO 488 (green line) and collagenase-ATTO 740 (red line) functionalization. (B) CD spectra of bare collagenase (blue line), 488 (green line) and 740 (pink line) conjugated enzyme at $0.5 \mu \mathrm{M}$, TRIS buffer $\mathrm{pH} 7.1$.

procedure described by Hermanson et al. ${ }^{27}$ The conjugation was evaluated by a UV-visible technique monitoring the signal at $280 \mathrm{~nm}$ related to the protein and the peaks at 488 (red line) and $740 \mathrm{~nm}$ (green line) associated with the dyes (Fig. 1A). A perfect degree of loading (DOL) equivalent to 2 for ATTO 488 and 1.67 for ATTO 740 were obtained and are reported in Table S1 (ESI $\dagger$ ).

Afterwards, with the aim of understanding whether any change in the structure of the enzyme had occurred, a CD spectroscopy analysis was performed. As expected, no variations between bare collagenase and conjugated enzyme were visible and in particular, as reported in Fig. 1B, all three samples showed a typical coiled structure with two pronounced minima at $220 \mathrm{~nm}$ and $210 \mathrm{~nm}$ and a maximum at $190 \mathrm{~nm} .^{30}$ A slight decrease in the Cotton effect was reported for ATTO 740 and this is probably due to the conjugation reaction and consequent changes in protein solubility; ATTO 740 is reported to be less hydrophilic than ATTO 488. Similar results, confirming our hypothesis, were obtained by evaluating the residual enzymatic assay in which collagenase-ATTO 488 revealed a high percentage of activity equal to $70.26 \pm 0.2 \%$, while the ATTO 740-conjugated protein scored only $9.17 \pm 2.1 \%$ (Table S1, ESI $\dagger$ ).

2.1.1 Synthesis of collagenase-ATTO 488 MPs and their structural/morphological characterization. Collagenase-ATTO 488 MPs were synthesized by the double emulsion technique with the addition of a porogenic agent known as ammonium bicarbonate (ABC), (Fig. S1A, ESI $\dagger$ ) in order to enhance the microparticle porosity and to ensure a faster release compared to a preparation without porogens (Fig. S1B, ESI $\dagger$ ). After washing steps and lyophilisation, the particles were suspended and their size was verified by a laser diffraction particle size analyzer, showing a size distribution with a mean diameter of $13.23 \pm 9.53 \mu \mathrm{m}$ (Fig. 2A).

The greater porosity of collagenase ABC-MPs than microparticles prepared following standard protocols was assessed by SEM microscopy (Fig. 2B and Fig. S2A, ESI $\dagger$ ). These data were confirmed by in vitro release studies, following the

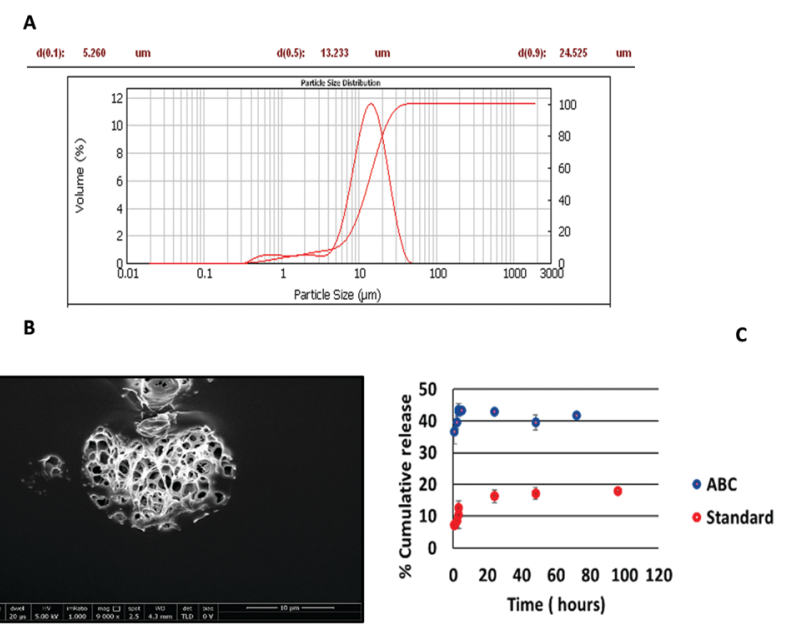

Fig. 2 Morphological characterization of collagenase MPs. (A) SEM microscopy of ABC MPs. (B) In vitro release studies of both MPs in TRIS buffer $\mathrm{pH} 7.1$ at $37^{\circ} \mathrm{C}$ at different time points. (C) MP size distribution analysis.

fluorescence emission of collagenase-ATTO 488, in which a faster rate of drug progression of $39.57 \pm 2.29 \%$ was reached in $2 \mathrm{~h}$ when the microparticles were prepared with ABC (Fig. 2C, blue circles) whereas in the case of the standard formulation at the same time the release was around $1 / 3(8.45 \pm 1.98 \%)$.

In the case of the $\mathrm{ABC}$ formulation, the last time point with an appreciable fluorescence signal is at $72 \mathrm{~h}$, whereas for the standard formulation it is at $96 \mathrm{~h}$, since the release is slower.

An excellent entrapment efficiency $(93.43 \pm 4.0 \%)$ calculated after complete disruption of the microparticles and fluorescence measurement of the solutions $\left(\lambda_{\mathrm{exc}}=488 \mathrm{~nm}, \lambda_{\mathrm{em}}=\right.$ $502 \mathrm{~nm}$ ) was reached with the addition of the $\mathrm{ABC}$ into the formulation (Table S2, ESI $\dagger$ ) and these results were confirmed by confocal images in which ABC-MPs showed a very high and homogeneous fluorescence signal related to the protein within the porous structures (Fig. 3A).

The standard formulation exhibited an entrapment efficiency of just $23.86 \pm 1.9 \%$. The increase in the encapsulation 

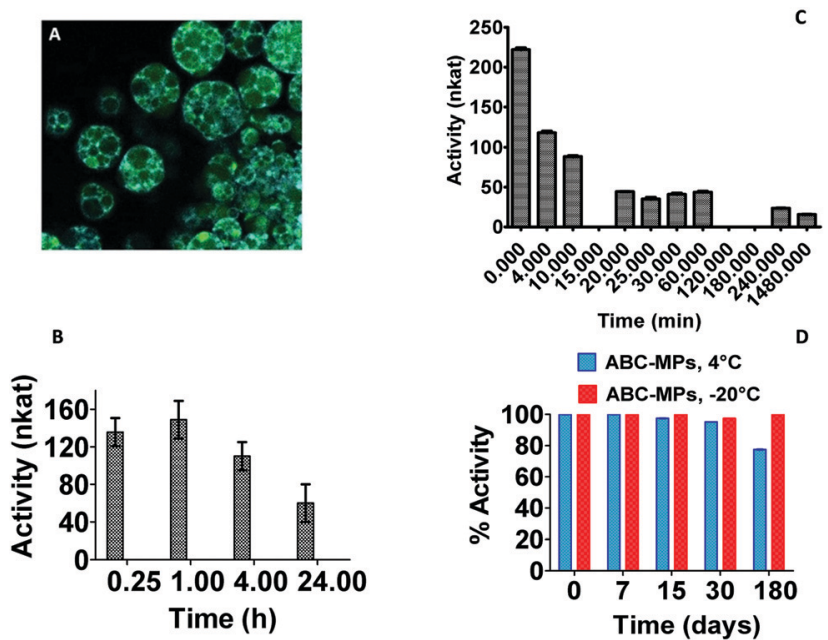

Fig. 3 (A) Confocal microscopy of ABC-MPs. Fluorescence images were acquired using a $\lambda_{\text {exc }}$ of $488 \mathrm{~nm}$ and a $\lambda_{\text {emiss }}$ between 500 and $600 \mathrm{~nm}$. Cyan blue is related to PLGA acquired in DAPI range. (B and C) Activity assay of (B) collagenase released from MPs and (C) free enzyme at different time points after incubation at $37{ }^{\circ} \mathrm{C}$. (D) Stability assay of embedded enzyme at 4 and $-20{ }^{\circ} \mathrm{C}$.

efficiency in the ABC procedure (Table S2 and Fig. S2-B, ESI $\dagger$ ) may be due to a favourable protein-polymer interaction at the interface due to the basic $\mathrm{pH}(\mathrm{pH} 7.5)$ with respect to the standard emulsion $(\mathrm{pH}=5.01$, near the $\mathrm{pI}$ of the protein). Indeed, basic $\mathrm{pH}$ modifies the protein charge distribution and subsequently its hydrophobicity, resulting in a more negatively charged surface and consequently a greater partitioning of collagenase in the aqueous phase is favoured. It is well known that near their $\mathrm{pI}$ value, proteins should be mostly neutral, and therefore rather hydrophobic and so less prone to be incorporated into a water phase. ${ }^{31,32}$

In order to verify the effects of the synthetic procedures on the collagenase bioactivity, enzymatic activity studies were carried out on both collagenase discharged after MP disruption and on protein released over time by the microparticles. After the encapsulation procedure, ABC-MPs kept the protein active at $44 \pm 1.20 \%$, but excellent results were obtained by analyzing the enzyme released from MPs at different time points (15 min to $24 \mathrm{~h}$ ) subsequent to their incubation at $37^{\circ} \mathrm{C}$. Indeed, as shown in Fig. 3B, our formulation is able to guarantee an activity of the enzyme $\geq 100$ nkat $(74.62 \%)$ up to $4 \mathrm{~h}$, reaching the specification for injectable formulations, ${ }^{33}$ unlike the free enzyme that has already lost $80 \%$ ( $\leq 44.4$ nkat) of its activity after 20 min (Fig. 3C). Regarding the stability of the enzyme inside MPs, the activity was measured up to 6 months and, as displayed in Fig. 3D, MPs are capable of storing protein activity close to 70 and $100 \%$ when kept at $4{ }^{\circ} \mathrm{C}$ or $-20{ }^{\circ} \mathrm{C}$, respectively. These data underline the impact of our polymeric structures in avoiding protein degradation, providing a powerful tool to produce stable pharmaceutical preparations.

\subsection{In vitro collagenase-MP activity in 3D collagen model}

In order to confirm the proteolytic activity of collagenase after the entire process of encapsulation, in vitro experiments, using a 3D collagen model (developed as reported in the Experimental section), were performed by multiphoton microscopy through second harmonic generation (SHG) analysis. SHG is a well-established and powerful technique to quantify collagen 3D organization in tissues and in particular in skin dermis. SHG signals are generated by collagen hierarchical organization in a triple helix, which are improved when collagen molecules form fibrils, while they decrease if the molecular architecture is destroyed. ${ }^{34,35}$ In our experiment, a collagen 3D model was incubated with collagenase released from MPs and SHG signals were recorded from 0 to $1500 \mathrm{~s}$. The control was performed by incubating collagen with TRIS buffer. As can clearly be seen from Fig. 4, the SHG signal relating to the treated collagen was significantly decreased compared to the untreated sample $(0.9697 \pm 0.01400$ vs. $0.6937 \pm 0.1047$; $p<0.0310)$ where it was almost constant over time. These data were also confirmed by Z-stack acquisition of both samples. Fig. S3 (ESI $\dagger$ ) clearly shows a decreasing signal and a nonhomogeneous structure for the treated collagen (Fig. S3-A, ESI $\dagger$ ) compared to the control (Fig. S3-B, ESI $\dagger$ ).

\subsection{Production and characterization of bi-compartmental polymeric microneedles}

After MP synthesis, we proceeded with a stamp-based method to fabricate bi-compartmental polymeric microneedles. Fig. 5A-F show an overview of the whole fabrication process. Briefly, a PDMS stamp with conical cavities is first filled with a solution of fast dissolvable polymers by drop-casting; then, after drying, the remaining parts of the cavities are filled with PLGA-based MPs which are pressed and plasticized by using a non-invasive soft plasticization method. ${ }^{20}$

Finally, the array of microneedles is harvested by a plasticized layer of PVP (polyvinylpyrrolidone) deposited onto a poly(methyl methacrylate) PMMA substrate. In more detail, the PDMS stamp is obtained by a master of microcones designed to produce microneedles sufficiently long to penetrate across the stratum corneum barrier and be inserted into the skin without pain. ${ }^{36}$ Microcones were produced with $600 \mu \mathrm{m}$ height, $300 \mu \mathrm{m}$ diameter base and $600 \mu \mathrm{m}$ center-center distance, for a total of 256 microcones per $\mathrm{cm}^{2}$ (Fig. 6A). Compared to a

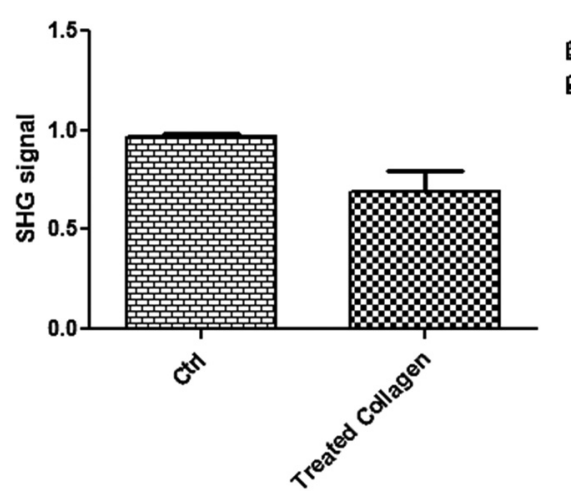

国 Ctrl $\mathbf{\infty}$ Treated Collagen

Fig. 4 SHG signals of control and treated collagen; the difference between samples was significant ( $p<0.0310, n=5$ ). 

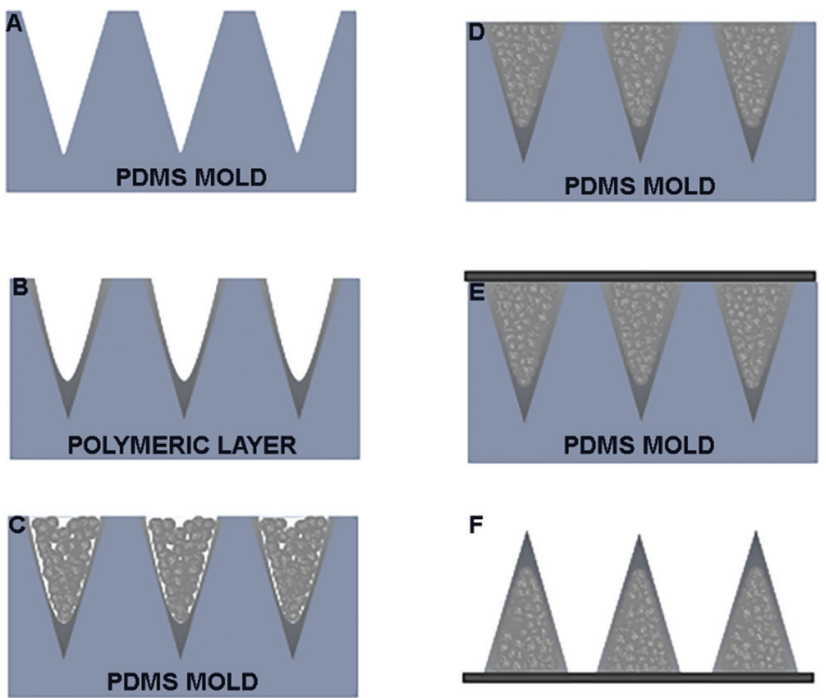

Fig. 5 (A) PDMS mold. (B) Deposition of polymeric solution on PDMS mold. (C) Dried layer. (D) MNs filled with MPs. (E) Compacted and HL applied. (F) MNs extracted from mold. MPs, which are pressed and plasticized by using a non-invasive soft plasticization method previously reported. ${ }^{19}$

previous study, ${ }^{20}$ the $25 \times 0.8$ NA oil-immersion objective of a Nanoscribe system was used to reduce the fabrication time of the master up to 15 times as compared to the $63 \times 1.4$ NA oilimmersion objective. The lower objective resolution provides high printing speed and, simultaneously, the realization of tips with a radius of curvature (set at $5.0 \mu \mathrm{m}$ ) (Fig. 6B) according to the specifications reported in the literature to break the stratum corneum $(\leq 25 \mu \mathrm{m}) .{ }^{37}$ To further increase the master fabrication speed (almost 4 times), microcones were designed with an external shell internally reinforced by a hollow scaffold; only these structures were photo-polymerized by $2 \mathrm{PP}$ whereas the liquid photoresist inside the microcone was cured afterwards using a

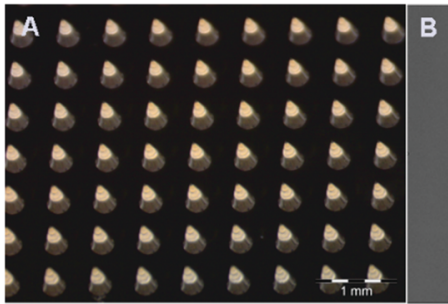

\section{B}
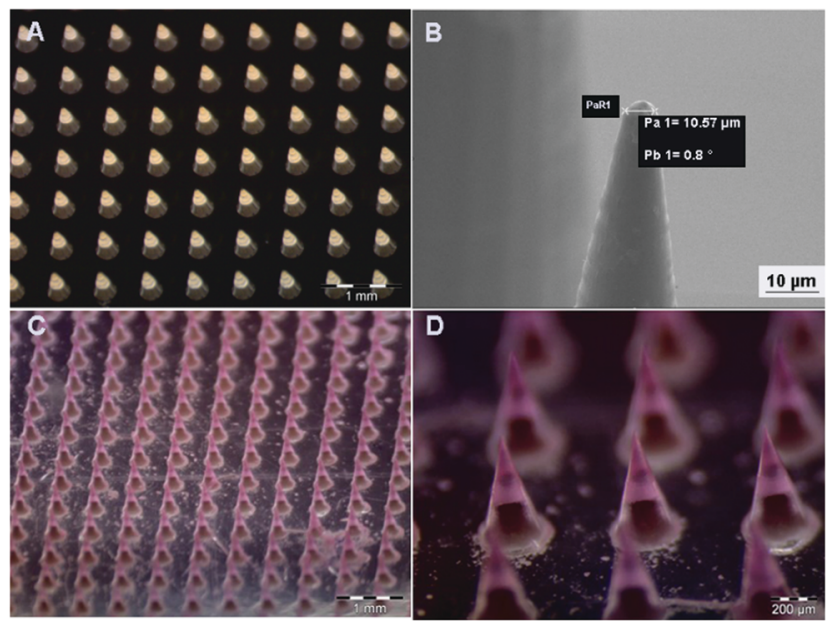

Fig. 6 (A) Stereomicroscope micrographs of master fabricated using 2-photon polymerization. (B) SEM micrograph of the master tip. (C and D) Stereomicroscope micrographs of microneedle patches with rhodamineloaded tips.
UV lamp. The master fabrication by $2 \mathrm{PP}$ using the Nanoscribe system is the most expensive step of the commonly used processes because of the printing time and the fragility of the cured material. To reduce the amount of masters produced and to guarantee the superior duration of the original master, low-cost NOA 60 replica masters were fabricated so that many replicas of the negative PDMS can be obtained from them. Negative micromolds were produced using the biocompatible, flexible, and nontoxic PDMS that, thanks to its durability, gives the possibility of producing tens of microneedle patches without being damaged or losing the shape of the cavities. PDMS micromolds can be reused by cleaning them with water or ethanol (for tip residues), and Scotch tape (for MP residues); also, PDMS allows high-definition replicas. $^{38,39}$ Because of the high hydrophobicity of PDMS, a low amount of surfactant (Tween 20) was added to the polymer solution to reduce surface tension and promote the spread of the drop/solution dispensed on the micromold. ${ }^{40}$ Mechanical strength is a fundamental feature required for microneedle application. To make this possible, PVP was selected as the structural material of the microneedle tips. It contains rigid rings in its chemical backbone that makes the polymer mechanically strong. Additionally, PVP has high water solubility at room temperature, which facilitates rapid dissolution once inserted into the skin, and no organic solvents are needed to fill the micromold. ${ }^{41}$ In this work, hyaluronic acid (HA) was mixed with the PVP solution to make the dried polymer layer more tensile than just PVP, even in the case of strong dehydration which is beneficial for the encapsulation of labile molecules. The other advantage of adding HA was to increase the dissolving time of the tips from a few seconds (just PVP) to a few minutes, preventing the possibility of losing the tip shape very quickly once in contact with the skin, which may affect the penetration ability. The addition of HA was also useful for the subsequent MP filling step that requires mechanical pressure on the stamp, which may fracture the underlying material. Furthermore, HA is a particularly attractive material for biomedical applications being an important component of the extracellular matrix (ECM) of the human body. ${ }^{42}$ As compared to the previous work, ${ }^{20}$ due to the increase in viscosity of the PVP-HA solution, spin coating was replaced with the drop-casting technique. This technique is advantageous because (1) it is an easily-tunable process, (2) it avoids wastage of materials, requiring low-cost and common tools, as dispenser and desiccator. The MP filling procedure and harvesting followed the already reported procedure. ${ }^{20}$ Due to the hygroscopic nature of $\mathrm{PVP}^{43}$ and $\mathrm{HA},{ }^{44}$ the fabricated $\mathrm{MN}$ patches were kept in an environment with low values of relative humidity in order to avoid moisture absorption by HA and PVP leading to a reduction in their mechanical strength. Therefore, each $\mathrm{MN}$ was sealed and stored in a desiccator at room temperature $\left(25^{\circ} \mathrm{C}\right)$ in the presence of dehydrating salts. Fig. $6 \mathrm{C}$ shows the uniformity in terms of tip sharpness of microneedle patches produced with this technique. To verify the complete filling of the microneedle body with MPs, microparticles loaded with a fluorophore (rhodamine) were prepared and used. The uniformity of fluorescence confirms the homogenous distribution of MPs within the microneedle body (Fig. 6D). Moreover, as shown in Fig. S4A and B (ESI $\dagger$ ), the 
embedded MPs retained their characteristics in terms of microstructure, demonstrating that the microneedle production method does not affect MP morphology.

\subsection{Microneedle release test into an in vitro dermis equivalent}

In order to test the microneedle release inside the dermis model (see the Experimental section), an MN patch was indented in the collagen and the enzyme fluorescence intensity from both MN compartments was followed over time, through the in vitro model volume by means of confocal microscopy. For this experiment, the microneedle tip was loaded with collagenase-ATTO 647 (that gives a signal displayed in red - conjugated in our lab, data not shown), while the MPs were loaded with collagenase-ATTO 488 (that gives a signal displayed in green). The experiment was performed for both MNs loaded with the standard MPs and MNs loaded with the ABC-MPs. In particular, three sampling times were chosen: $30 \mathrm{~min}, 2 \mathrm{~h}$ and $24 \mathrm{~h}$. At each time point, the images of the $z$-position at the cone base and at the half height of the microneedles were acquired. In the following, the latter positions are called top and middle view, respectively. In more detail, the top view shows what is happening to the collagen in contact with the base of the patch, while the middle view shows what is happening to the collagen at half of the height of the microneedles. Moreover, the images show the orthogonal section of the selected $z$-position, giving information about the $y-z$ and $x-z$ planes. For each $z$-position, the green channel, the red channel and the merging of the channels were acquired.

At first, the standard-MP loaded MNs were indented in the in vitro dermis model. $30 \mathrm{~min}$ after the indentation, the images show that, at both collagen sections, the predominant signal comes from the enzyme inside the fast-dissolving tip (red). Moreover, a slight accumulation of MPs (i.e. green signal) in the upper sections (see top view) of the collagen is also visible (Fig. 7A-G; see Fig. S5 for the split channels, ESI $\dagger$ ). After $2 \mathrm{~h}$, the images show an increment in the signal coming from the enzyme inside the MPs (green), both in the top and middle sections of collagen (Fig. 7B-H; see Fig. S6 for the split channels, ESI $\dagger$ ) compared to Fig. 7A-G. Finally, after $24 \mathrm{~h}$ at both collagen sections, the predominant signal comes from the enzyme inside the slow-dissolving compartment of the needles, the MPs (green). Moreover, a decay in the red signal is also visible in both collagen sections, probably caused by the inevitable signal attenuation due to the protein diffusion and dilution into the whole collagen volume, especially after $24 \mathrm{~h}$ (Fig. 7C-I: see Fig. S7 for the split channels, ESI $\dagger$ ).

Then, the ABC-MP loaded MNs were indented in the in vitro dermis model. $30 \mathrm{~min}$ after the indentation, images show that at both collagen sections the predominant signal comes from the enzyme inside the fast-dissolving tip (red). Moreover, a considerable amount of protein in the upper sections of collagen is also visible (Fig. 7D-J; see Fig. S8 for the split channels, ESI $\dagger$ ). In this case, the signal coming from the MPs in the top view is higher ( $+43 \%$ ) compared to Fig. $7 \mathrm{~A}-\mathrm{G}$, giving a proof of the presence of a higher amount of enzyme inside the ABC-MPs compared to the standard-MPs. After $2 \mathrm{~h}$, the images

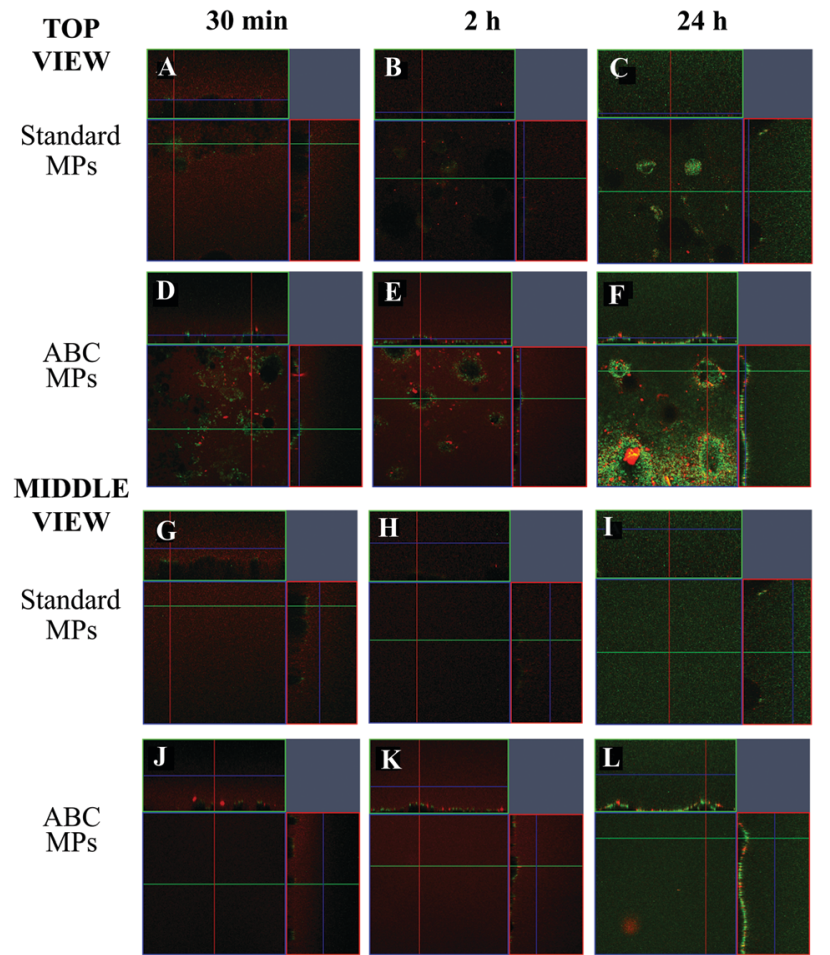

Fig. 7 Release test of $600 \mu \mathrm{m}$ microneedles loaded with the standard vs. ABC-MPs in the in vitro skin equivalent model after $30 \mathrm{~min}$ : (A) and (D) top view and $(G)$ and $(J)$ middle view; after $2 \mathrm{~h}$ : $(B)$ and $(E)$ top view and $(H)$ and (K) middle view; after $24 \mathrm{~h}$ : (C) and (F) top view and (I) and (L) middle view. Images show the merging of the green and red channels.

show an improvement in the signal coming from the enzyme inside the microparticles (green), for both the upper and middle sections of collagen (Fig. 7E-K; see Fig. S9 for the split channels, ESI $\dagger$ ) compared to Fig. 7D-J. Even in this case, the signal coming from the MPs in both views is higher (+33\%) compared to Fig. 7B-H, confirming again the presence of a higher amount of enzyme in the ABC-MPs. Finally, after $24 \mathrm{~h}$, the images show that at both collagen sections the predominant signal comes from the enzyme inside the slow-dissolving compartment of the needles, the MPs (green) (Fig. 7F-L; see Fig. S10 for the split channels, ESI $\dagger$ ). A decay in the red signal is also visible in both collagen sections, as observed in the previous case. Once more, the signal coming from the MPs, both in the top and middle views, is broadly higher $(+115 \%)$ compared to Fig. 7C-I.

\subsection{Preliminary in vivo indentation tests by photoacoustic evaluation of fluorescent collagenase-ATTO 740 delivered by microneedle tips}

In order to confirm the in vitro results on a dermis model, with regard to the ability of microneedles to cross the stratum corneum, their mechanical strength and their capability to release the encapsulated drug, a preliminary in vivo test of the microneedle patch was performed on rat epidermis. In detail, collagenase-ATTO 740 was loaded into the tip of the microneedles and the patch was applied to the skin surface. 
The relative photoacoustic signal of fluorescent collagenase was detected and quantified at different time points from 0 to $90 \mathrm{~min}$ after $3 \mathrm{~min}$ of application. The obtained results clearly indicated good microneedle indentation (Fig. 8A) showing good photoacoustic signals in all analyzed time periods with a consistent signal up to $90 \mathrm{~min}$ (Fig. 8B).

\section{Experimental section}

Poly(lactic-co-glycolic acid) (PLGA) RESOMER ${ }^{\circledR}$ RG $504 \mathrm{H}$, 38 000-54 000 Dalton, lactide : glycolide $=50: 50$, was purchased from Boeringer Ingelheim. Dichloromethane (DCM), ammonium bicarbonate $(\mathrm{ABC})$, citric acid monohydrate, dimethyl sulfoxide (DMSO), tris(2-carboxyethyl)phosphine hydrochloride (TCEP), sodium sulfate anhydrous $\left(\mathrm{NaSO}_{4}\right)$, ethyl acetate, methanol (MetOH), trizima base (TRIS), calcium chloride $\left(\mathrm{CaCl}_{2}\right)$, hydrochloric acid $(\mathrm{HCl})$, sodium dodecyl sulfate (SDS), sodium hydroxide $(\mathrm{NaOH})$, Mowiol $^{\circledR}$ 40-88 (poly(vinyl) alcohol, PVA, MW 27 000-32000 PVA) were purchased from Sigma-Aldrich. Double-distilled water was pretreated with a Milli-Q R Plus System (Millipore Corporation, Bedford, USA). ATTO 488 and 740 maleimide were purchased from ATTO-TEC. Collagenase and collagenase substrate (Wünsch substrate, PZ-L-Pro-L-Leu-Gly-L-Pro-D-Arg) were kindly offered by FIDIA Farmaceutici. Polyvinylpyrrolidone (PVP $856568 M_{\mathrm{w}} 55 \mathrm{KDa}$ ), TWEEN $^{\circledR}$ 20, dimethyl carbonate (DMC, D152927), sulforhodamine B (Rhod) (SulphoRh6G, S470899) were purchased from Sigma-Aldrich. Hyaluronic acid $\left(M_{\mathrm{w}} 200 \mathrm{KDa}\right)$ was provided by Fidia $^{\circledR}$. NOA 60 glue was purchased from Norland Optical Adhesive. Poly(dimethylsiloxane) (PDMS) was provided by Sylgard R (184 Silicone Elastomer Kit, Dow Corning). Poly(methyl methacrylate) (PMMA) was purchased from GoodFellow. IP-S negative tone photoresist and ITO-coated glass substrate were purchased from Nanoscribe GmbH. Developer mr-Dev 600 was purchased from Micro Resist Technology GmbH.
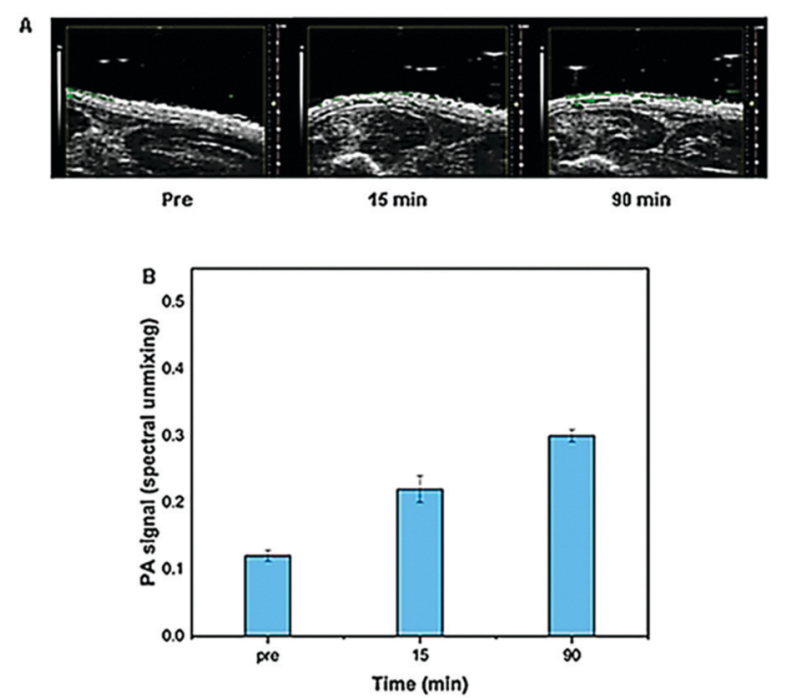

Fig. 8 In vivo photoacoustic signals of collagenase-ATTO 740 MNs (A) before and after (15 and $90 \mathrm{~min}$ ) and (B) their quantification.

\subsection{Collagenase functionalization with ATTO 488 and ATTO} 740 and purification

Collagenase was functionalized with ATTO 488 and ATTO 740 fluorophores using a cysteine-maleimide coupling. In detail, a solution of collagenase of $2 \mathrm{mg} \mathrm{mL}^{-1}$ was firstly reduced for $1 \mathrm{~h}$ with a 4 -fold molar excess of TCEP $(0.1 \mathrm{M})$, and afterwards a 20 -fold molar excess of ATTO 488/740 was added. The reaction was then incubated at $25{ }^{\circ} \mathrm{C}$ for $2 \mathrm{~h}$ under gentle shaking. The excess of fluorophores was removed using a 3000 Dalton Amicon Ultra-4 centrifuge filter. The enzyme-dye conjugation was validated by the acquisition of UV-visible (UV-vis) spectra (Cary 100 Scan Spectrophotometer, Agilent).

\subsection{Circular dichroism (CD) spectroscopy}

CD spectra were recorded on a Jasco J-1500 spectropolarimeter (J-1500-150, Japan) in a $1.0 \mathrm{~cm}$ path-length quartz cell. CD spectra were registered at $25{ }^{\circ} \mathrm{C}$ in the far UV region using a concentration of $0.5 \mu \mathrm{M}$ both for the conjugated and the bare enzyme. The spectra were obtained with an average of 3 scans by subtracting them from blank samples, as previously described. ${ }^{45-54}$

\subsection{Collagenase-ATTO $488 \mathrm{MP}$ production}

The collagenase-ATTO 488 MPs were prepared by water/oil/ water $(\mathrm{W} / \mathrm{O} / \mathrm{W})$ double emulsion/solvent evaporation. In particular, collagenase-ATTO 488 MPs were prepared with and without a porogenic agent (Fig. S1A and B, ESI $\dagger$ ) according to a previously reported method used for curcumin. ${ }^{13}$ The internal water phase, composed of $100 \mu \mathrm{L}$ of collagenase-488 at a concentration of $0.5 \mathrm{mg} \mathrm{mL}{ }^{-1}$ was homogenized with $100 \mathrm{mg}$ of PLGA in $1 \mathrm{~mL}$ of DCM by an Ultra-Turrax ${ }^{\circledR}$ T25 basic, IKA ${ }^{\circledR}$ WerkeDigital High-Speed Homogenizer System, at $20000 \mathrm{rpm}$ for $30 \mathrm{~s}$. In the case of using a porogenic agent, an intermediary step was developed: $100 \mu \mathrm{L}$ of a gas foaming $\mathrm{ABC}$ porous agent solution $\left(7.5 \mathrm{mg} \mathrm{mL}^{-1}\right)$ was homogenized with the first emulsion $\mathrm{W} / \mathrm{O}$ at $20000 \mathrm{rpm}$ for $30 \mathrm{~s}$.

Then the first emulsion with and without $\mathrm{ABC}$ was homogenized for $1 \mathrm{~min}$ at $25000 \mathrm{rpm}$ into a water phase formed by $10 \mathrm{~mL}$ of $2 \%(\mathrm{w} / \mathrm{v})$ PVA solution. Finally, the second emulsion was poured in $40 \mathrm{~mL}$ of Milli-Q water under mechanical stirring for $3 \mathrm{~h}$ at $450 \mathrm{rpm}$ (Heidolph RZR 2102 control) to allow DCM diffusion and evaporation. All the steps were performed by keeping the samples in an ice bath to preserve the protein activity during the whole procedure.

As to washing steps: the MPs were collected, washed three times with Milli-Q water by centrifuging at $10000 \mathrm{rpm}$ for $10 \mathrm{~min}$ at $4{ }^{\circ} \mathrm{C}$ (SL16R Centrifuge, Thermoscientific, USA), lyophilized overnight (HetoPowerDry PL6000 Freeze Dryer, Thermo Electron Corp., USA; $-50{ }^{\circ} \mathrm{C}, 0.73 \mathrm{hPa}$ ) and stored at $-20{ }^{\circ} \mathrm{C}$ with desiccating agents until further investigation.

\subsection{Collagenase-ATTO $488 / 740$ and collagenase-ATTO 488 MP activity assay}

The activity (expressed as nkat) of collagenase-ATTO 488/740 and collagenase-ATTO 488 MPs was quantified following the 
Wünsch assay. ${ }^{9}$ In particular, three batches of each sample were incubated with the collagenase chromophore substrate for $15 \mathrm{~min}$ and the resulting peptide, formed upon collagenase activity, was analyzed by UV-Vis at $320 \mathrm{~nm}$. Data were normalized compared to the concentration of the proteins used and the weight of MPs and expressed as a percentage.

The activity assay was also evaluated for 6 months in order to study the stability of collagenase inside MPs.

\subsection{Collagenase-ATTO 488 inside MP encapsulation efficacy (\% $\eta)$}

The $\% \eta$ of collagenase-ATTO 488 inside MPs was evaluated by dissolving $10 \mathrm{mg}$ of MPs or 3 patches of MP-MNs in a solution of $50 \% \mathrm{DMSO} / 0.5 \% \mathrm{SDS} / 0.1 \mathrm{M} \mathrm{NaOH}$ for $1 \mathrm{~h}$ under vigorous stirring. The collagenase content was analyzed by fluorescence, following the signal of collagenase-ATTO 488 at $\lambda_{\mathrm{em}}=504 \mathrm{~nm}$ and using a titration curve of the free collagenase-ATTO 488 made at the same time and under the same conditions. Each experiment was performed in triplicate. The average $\% \eta$ was calculated as (actual drug loading/theoretical drug loading) $\times 100 \%$.

\subsection{Collagenase-ATTO $488 \mathrm{MP}$ in vitro release study}

In vitro drug release studies were carried out by suspending $10 \mathrm{mg}$ of collagenase-ATTO 488 MPs or 3 patches of MPS-MNs in $1.5 \mathrm{~mL}$ of TRIS buffer. Samples were incubated at $37^{\circ} \mathrm{C}$ and shaken under gentle conditions. At fixed time points $(30 \mathrm{~min}$, $1 \mathrm{~h}, 2 \mathrm{~h}, 3 \mathrm{~h}, 4 \mathrm{~h}, 24 \mathrm{~h}$ and $48 \mathrm{~h}$ ), $1 \mathrm{~mL}$ of sample was withdrawn after MP sedimentation using centrifugation for $15 \mathrm{~min}$ at $10000 \mathrm{rpm}$ (MicroCL21R, Centrifuge, Thermoscientific, USA) and analyzed by fluorescence, as previously reported. The pellet was re-suspended in the same volume of fresh buffer. A titration curve of the free collagenase-ATTO 488 was performed under the same conditions. All the tests were executed in triplicate. The average \%release was calculated as (actual drug loading/theoretical drug loading) $\times 100 \%$.

\subsection{Fabrication of master and micromold}

A Nanoscribe Photonic Professional GT system (Nanoscribe $\mathrm{GmbH}$ ) was used to fabricate the microcone array master by means of a 2-photon polymerization (2PP) technique, according to a method previously reported. ${ }^{20}$ Briefly, the Nanoscribe system uses a $780 \mathrm{~nm}$ Ti-sapphire laser emitting $\approx 100 \mathrm{fs}$ pulses at $80 \mathrm{MHz}$ with a maximum power of $150 \mathrm{~mW}$. An ITO-coated glass substrate (Nanoscribe $\mathrm{GmbH}$ ) with an IP-S negative photoresist was placed in a holder and inserted into a piezoelectric $x / y / z$ stage. To guarantee a high writing speed from the laser, a galvo stage was used which allows a fabrication process 100 times faster than the piezo mode setup. This time, a $25 \times$ 0.8 NA oil immersion objective was used instead of a $64 \times$ 1.4 NA objective, in order to further enhance the fabrication speed (almost 10 times).

As reported in the previous protocol, a $16 \times 16$ microcone array was fabricated in a conical shape with a microcone height of $600 \mu \mathrm{m}$, a base diameter of $300 \mu \mathrm{m}$ and a center-to-center interval of $600 \mu \mathrm{m}$. The fabricated master was immersed in developer solution mr-Dev 600 for $30 \mathrm{~min}$ in order to remove the uncured negative photoresist and then washed with IPA.
Finally, to enhance the microcone stiffness, the master was put under a UV lamp (16 $\left.\mathrm{mW} \mathrm{cm}^{-2}\right)$ for $4 \mathrm{~h}$. Then, the negative micromolds used for the production of the microneedle patches were fabricated by a replica molding technique. A solution of liquid PDMS precursor and its curing agent $(10: 1 \mathrm{w} / \mathrm{w})$ was dispensed on the master and then put under a vacuum to remove entrapped air bubbles; the PDMS solution was cured in an oven at $80{ }^{\circ} \mathrm{C}$ for $2 \mathrm{~h}$ and peeled off after cooling to RT. ${ }^{55}$ To preserve the original master from wearing out and tips breaking in the fabrication of the negative PDMS molds, a replica master was fabricated using NOA 60 photosensitive polymer. Liquid NOA 60 was poured onto the PDMS micromold, degassed under vacuum for $2 \mathrm{~h}$ and cured for 20 min under a UV lamp (wavelength $365 \mathrm{~nm}$ ). Then the positive NOA replica master was extracted and attached to a Petri dish for further PDMS micromold replicas.

3.7.1 Production of the microneedle patch. The dissolving microneedle patch was fabricated using the drop casting technique for the production of the tips and manual filling for the internal body. In detail, polyvinylpyrrolidone (PVP), hyaluronic acid (HA), and Tween 20 were dissolved in distilled water with the following percentages (w/w): 5.9, 1.5, 0.2;0.1 $\mathrm{mg} \mathrm{mL}^{-1}$ of FITC was mixed into the solution for fluorescence microscopy. $150 \mu \mathrm{L}$ of aqueous solution was cast onto the PDMS micromold, degassed for $30 \mathrm{~min}$ in a desiccator, and dried at room temperature. Then, to complete the microneedles, the previously reported method was used. ${ }^{19}$ In brief, PLGA MPs were incorporated into the cavities by the aid of a spatula under an optical stereomicroscope (Olympus, SZX16 double objective). Then, an array of PMMA pillars was pressed onto the PDMS micromold in order to compact the MPs inside the cavities. Subsequently, the MPs were slightly plasticized using a mixture of ethanol $(4 \mathrm{~mL})$ and DMC $(0.5 \mathrm{~mL})$ for $6 \mathrm{~min}$. To extract the MN patch, $200 \mu \mathrm{L}$ of $25 \%$ (w/v) PVP aqueous solution was dispensed on a rectangular piece of PMMA (0.5 mm thickness), which had previously undergone oxygen plasma treatment for 1 min to increase the adhesion between PVP solution and PMMA, and dried on a hot plate at $40{ }^{\circ} \mathrm{C}$ for $2 \mathrm{~h}$. Then, the PVP harvesting layer was plasticized for 11 min under the previously reported conditions and placed upon the micromold. After 30 min of drying at RT, the microneedle patch was extracted.

This time, microneedle patches were kept in aluminiumlaminated sachets containing Tri-Sorb molecular sieves and stored in a desiccator at room temperature $\left(25^{\circ} \mathrm{C}\right) .{ }^{36}$

\subsection{MN and MP characterization}

3.8.1 Optical stereomicroscopy. Microneedle masters fabricated by the Nanoscribe system were analyzed by a stereomicroscope (Olympus, SZX16 double objective) to ensure the good quality of the fabrication and integrity of the tips. After extraction, each microneedle patch was analyzed by the stereomicroscope to investigate the sharpness of the tips and the pattern distribution.

3.8.2 Microparticle size and polydispersity index (PdI). The MP size distribution was evaluated by static light scattering 
(Mastersizer 2000, Malvern Instruments, Malvern, UK) using a water solution of $3 \mathrm{mg} \mathrm{mL}{ }^{-1}$ of MPs. ${ }^{54}$

3.8.3 Confocal microscopy. MPs inside the microneedle body were acquired by detecting PLGA auto-fluorescence in DAPI range. Sample morphology was investigated using a confocal microscope (Leica Microsystems TCS SP5 II, Germany) with a $20 \times$ air objective. Images were acquired with a resolution of $1024 \times 1024$ pixels. Collagenase-ATTO 488 MPs were characterized by confocal microscopy in order to evaluate the signal of the protein inside the porous structures. Fluorescence images were acquired using an HCX IRAPO L 40×/0.95 water objective with a $488 \mathrm{~nm}$ laser as $\lambda_{\text {exc }}$.

3.8.4 Scanning electron microscopy (SEM). The external and internal morphology of the MPs was studied by SEM microscopy, as already described. ${ }^{16,56}$ In detail, samples were attached onto a standard SEM pin stub, placed into a sputter coater which covered them with a $15 \mathrm{~nm}$ thickness gold layer and analyzed by FESEM at 15-25 kV using an SE2 detector. $100 \mu \mathrm{L}$ of MPs were deposited on a standard SEM pin stub, gold-sputtered (10 nm thickness) using a HR208 Cressington sputter coater and analyzed by FESEM ULTRA-PLUS (Zeiss) at $5 \mathrm{kV}$ with the SE2 detector. A $2 \mathrm{~mm}$ thickness PDMS, cured at $80{ }^{\circ} \mathrm{C}$ for $30 \mathrm{~min}$, was used to investigate the internal structure, as previously reported. ${ }^{13}$

\subsection{In vitro dermis equivalent}

The 3D in vitro dermis equivalent model was set up by embedding Human Dermal Fibroblast (HDF) cells in a collagen matrix. Cells were cultured with complete medium, composed of Eagle's minimal essential medium (EMEM) supplemented with $20 \% \mathrm{FBS}, 1 \%$ glutamine, $1 \%$ non-essential amino acids, and $100 \mathrm{U} \mathrm{mL}^{-1}$ penicillin, $100 \mathrm{mg} \mathrm{mL}^{-1}$ streptomycin. The cells were maintained in $100 \mathrm{~mm}$ diameter cell culture dishes in a humidified controlled atmosphere at $37{ }^{\circ} \mathrm{C}$ and $5 \% \mathrm{CO}_{2}$. The medium was changed every $2-3$ days. Collagen gels were prepared using rat-tail collagen solution (ibidi ${ }^{\circledR}$ ) and reconstituted according to the manufacturer's protocol and were used to test the in vitro activity of collagenase-MP. $6 \times 10^{4} \mathrm{HDF}$ cells were suspended in $300 \mu \mathrm{L}$ of $4 \mathrm{mg} \mathrm{mL}^{-1}$ collagen solution and poured into a Millicell ${ }^{\circledR}$ (cell culture inserts, Millipore). The system was then incubated at $37^{\circ} \mathrm{C}$ for $30 \mathrm{~min}$ to allow collagen fibrillogenesis and, afterwards, fresh cell culture medium was added to the gel.

\subsection{Release test into the in vitro dermis equivalent of microneedles}

In order to test the microneedle release inside the dermis model, a microneedle patch was indented in the collagen and the fluorescent signal from the enzyme was followed at different time points (i.e. $30 \mathrm{~min}, 2 \mathrm{~h}$, and $24 \mathrm{~h}$ ), through the in vitro model volume, by means of confocal microscopy. For this experiment, a microneedle tip was loaded with collagenaseATTO 647 conjugated in our laboratory (data not shown) (that gives a signal displayed in red), while the microparticles were loaded with collagenase-ATTO 488 (that gives a signal displayed in green). The test was performed for both MNs loaded with the standard MPs and MNs loaded with the ABC-MPs and followed at different time points (i.e. $30 \mathrm{~min}$, $2 \mathrm{~h}$, and $24 \mathrm{~h}$ ), by means of confocal microscopy. Images were obtained by confocal microscopy (Leica Microsystems TCS SP5 II, Germany) with a $10 \times$ water immersion objective and images were acquired with a resolution of $1024 \times 1024$ pixels. Moreover, a semi-quantitative analysis was performed on at least 5 images for each $z$-plane to obtain the mean fluorescence intensity of the protein for both the standard and ABC-MPs. Briefly, using Image software, the mean grey value (MGV) of the green channel was measured for each image. Using the obtained parameters, the percentage increase of the MGV of the green channel between the standard and ABC-MPs was calculated according to the following equation:

$\%$ of increment $=\frac{\text { MGVA BCMPs }- \text { MGV standard MPs }}{\text { MGV standard MPs }} \times 100$

\subsection{Collagenase-ATTO $740 \mathrm{MN}$ preliminary in vivo release}

3.11.1 Animal preparation, patch application and imaging. The day before imaging experiments, the Sprague Dawley rats (male, weight and age at arrival: 200-250 g, supplied by Envigo RMS SARL, Gannat, France) were anesthetized under 3-4\% Sevoflurane gas anaesthesia $\left(\mathrm{O}_{2} 95.0 \%\right)$ and the skin area to be treated with microneedles was shaved using a razor and then Veet cream. Then, the microneedle patch was applied on the shaved skin by finger pressure for $3 \mathrm{~min}$, following by a washing step to remove surface collagenase residue. All the procedures involving the animals were conducted according to national and international laws on experimental animals (L.D. 26/2014; Directive 2010/63/EU) and to the approved experimental protocol procedure (Authorization No. 730/2015-PR). No validated non-animal alternatives are known to meet the objectives of the study. During the housing period the animals were maintained in a ventilated cabinet at the Animal Facilities of CEIP, via Ribes 5, 10010 Colleretto Giacosa (TO).

In more detail, each animal was treated with patches loaded with collagenase-ATTO 740 in the needle tips and in particular a patch with $0.56 \mathrm{pmol}$ of fluorophore was applied for $3 \mathrm{~min}$ by finger. After patch application, photoacoustic images were acquired at different time points (0-90 $\mathrm{min})$.

3.11.2 In vivo photoacoustic imaging. The rat was placed inside the Vevo Lazr2100 at 15 and $90 \mathrm{~min}$. after patch application. An acoustic gel was used as a coupling agent between the ultrasound transducer and the skin. The ultrasound frequency was set at $21 \mathrm{MHz}$, the PA gain was $40 \mathrm{~dB}$, and the US gain was $18 \mathrm{~dB}$. Photoacoustic spectra were acquired using a wavelength ranging from $680 \mathrm{~nm}$ to $970 \mathrm{~nm}$ with a step size of $5 \mathrm{~nm}$, and a persistence (frame averaging) of 3 .

3.11.3 Data analysis. Graph Pad Prism 8 software (Graph Pad Software, San Diego, CA, USA) was used for statistical analysis. The significance of statistical differences was determined by an unpaired $t$ test. A two-tailed $p$ value equal to or less than 0.05 was considered statistically significant. For the in vivo test, regions of interest (ROIs) were selected by the operator 
over the acquired PAI images. The photoacoustic signal within the ROIs was determined automatically by the software and reported as a PA average.

\section{Conclusions}

Delivery systems meant for the controlled release of labile molecules need to be designed with the aim of preserving the molecule during both production, storage and application. Here, we have designed porous PLGA MPs able to promote the prolonged release of a labile biomolecule, collagenase, but in a time frame of a few hours in order to avoid long exposure time of the biomolecule to a destabilizing wet environment. It is indeed known that collagenase activity is time and dose dependent, depending on pharmaceutical compositions; ${ }^{57}$ in particular, an enzyme activity between 7 and 20 nkat is required for dry formulations, while a higher activity ( $\geq 120$ nkat) is necessary in injectable formulations where the enzyme, reconstituted in water, undergoes degradation problems in a few hours $(\leq 8 \mathrm{~h}) .{ }^{33}$ Our system is a dry formulation, which explores a wet environment when implanted in the derma; however, we guarantee an enzyme activity of $\geq 100$ nkat, almost reaching the specification for injectable formulations. Additionally, as compared to the free enzyme, which needs to be stored at $-20{ }^{\circ} \mathrm{C}$ or $-80{ }^{\circ} \mathrm{C}$, collagenase embedded in our MPs has maintained an activity close to $70 \%$ even if stored at $4{ }^{\circ} \mathrm{C}$ for 6 months.

Such MPs are assembled in the shape of bi-compartmental microneedles that present fast dissolvable tips and a body made of assembled MPs. Microneedle tips and bodies are produced in two separated steps; therefore, cargo-loaded MPs are processed in a dry state with no risk of premature release during the process. In comparison to the previously developed protocol, the polyvinylpyrrolidone (PVP) tip has been replaced with a less fragile PVP-hyaluronic acid (PVP-HA) mixture to allow strong dehydration, which is useful for preserving the protein during microneedle storage. Remarkably, all the used materials are FDA approved. Finally, with in vitro diffusion studies we assessed the ability of the MP-embedded microneedles to release the enzyme in a prolonged but short time (a few hours) compared to the single compartment ones that rapidly dissolve without the possibility of modulating the delivery of the payload. ${ }^{58}$ To conclude, a preliminary in vivo test confirmed the ability of the microneedles to by-pass the stratum corneum of a rat and transfer collagenase into the skin.

These results suggest that tuning PLGA MP microstructure and using such MPs as building blocks for the fabrication of microneedles, in combination with fast dissolvable tips, may represent a promising technology to fine tune the controlled release of therapeutic labile compounds into the skin for a range of possible applications in healthcare.

Of course, when dealing with microneedles, sterilization, manufacturing/packaging and storage are principal factors to be taken into account for scaling up MNs before commercialization. Another issue is to make the application of microneedles user friendly. Therefore, we are now working on a strategy to make the microneedle patch co-planar to the skin before implantation without the use of applicators.

\section{Funding}

This work was supported by Fidia Farmaceutici S.p.A., via Ponte della Fabbrica 3/A - 35031 Abano Terme (PD), Italy.

\section{Conflicts of interest}

There are no conflicts to declare.

\section{Acknowledgements}

The authors thank Dr Valentina Mollo for her assistance in the acquisition of SEM images and Dr Fabio Formiggini for assistance in the acquisition of confocal images. The authors thank Fidia Farmaceutici S.p.A. for having supported the research, authorship and publication of this article.

\section{References}

1 M. Kesik-Brodacka, Progress in biopharmaceutical development, Biotechnol. Appl. Biochem., 2018, 65(3), 306-322.

2 S. Bhaskar and S. Lim, Engineering protein nanocages as carriers for biomedical applications, NPG Asia Mater., 2017, $\mathbf{9}(4)$, e 371.

3 S. La Manna, C. Di Natale, D. Florio and D. Marasco, Peptides as therapeutic agents for inflammatory-related diseases, Int. J. Mol. Sci., 2018, 19(9), 2714.

4 J. Renukuntla, A. D. Vadlapudi, A. Patel, S. H. Boddu and A. K. Mitra, Approaches for enhancing oral bioavailability of peptides and proteins, Int. J. Pharm., 2013, 447(1-2), 75-93.

5 S. Jacob, A. Shirwaikar, K. Srinivasan, J. Alex, S. Prabu, R. Mahalaxmi and R. Kumar, Stability of proteins in aqueous solution and solid state, Indian J. Pharm. Sci., 2006, 68(2), 154-163.

6 M. Vrkljan, T. M. Foster, M. E. Powers, J. Henkin, W. R. Porter, H. Staack, J. F. Carpenter and M. C. Manning, Thermal stability of low molecular weight urokinase during heat treatment. II. Effect of polymeric additives, Pharm. Res., 1994, 11(7), 1004-1008.

7 N. Washington, C. Washington and C. Wilson, Physiological pharmaceutics: barriers to drug absorption, CRC Press, 2000.

8 C. Peres, A. I. Matos, J. Conniot, V. Sainz, E. Zupančič, J. M. Silva, L. Graca, R. S. Gaspar, V. Preat and H. F. Florindo, Poly(lactic acid)-based particulate systems are promising tools for immune modulation, Acta Biomater., 2017, 48, 41-57.

9 H. K. Makadia and S. J. Siegel, Poly lactic-co-glycolic acid (PLGA) as biodegradable controlled drug delivery carrier, Polymers, 2011, 3(3), 1377-1397.

10 R. De Alteriis, R. Vecchione, C. Attanasio, M. De Gregorio, M. Porzio, E. Battista and P. A. Netti, A method to tune the 
shape of protein-encapsulated polymeric microspheres, $S c i$. Rep., 2015, 5(1), 1-9.

11 D. Klose, F. Siepmann, K. Elkharraz, S. Krenzlin and J. Siepmann, How porosity and size affect the drug release mechanisms from PLGA-based microparticles, Int. J. Pharm., 2006, 314(2), 198-206.

12 S. Mitragotri and J. Lahann, Physical approaches to biomaterial design, Nat. Mater., 2009, 8(1), 15-23.

13 C. Di Natale, V. Onesto, E. Lagreca, R. Vecchione and P. A. Netti, Tunable Release of Curcumin with an In SilicoSupported Approach from Mixtures of Highly Porous PLGA Microparticles, Materials, 2020, 13(8), 1807.

14 V. Sinha and A. Trehan, Biodegradable microspheres for protein delivery, J. Controlled Release, 2003, 90(3), 261-280.

15 J. Lademann, H. Schaefer, N. Otberg, A. Teichmann, U. Blume-Peytavi and W. Sterry, Penetration von Mikropartikeln in die menschliche Haut, Der Hautarzt, 2004, 55(12), 1117-1119.

16 R. Jamaledin, Di Natale, C. Onesto, V. Taraghdari, Z. B. Zare, E. N. Makvandi, P. Vecchione, R. Netti and P. A. Progress, Microneedle-Mediated Protein Delivery, J. Clin. Med., 2020, $\mathbf{9}(2), 542$.

17 A. Arora, M. R. Prausnitz and S. Mitragotri, Micro-scale devices for transdermal drug delivery, Int. J. Pharm., 2008, 364(2), 227-236.

18 N. Roxhed, B. Samel, L. Nordquist, P. Griss and G. Stemme, Painless drug delivery through microneedle-based transdermal patches featuring active infusion, IEEE Trans. Biomed. Eng., 2008, 55(3), 1063-1071.

19 R. Jamaledin, C. K. Yiu, E. N. Zare, L.-N. Niu, R. Vecchione, G. Chen, Z. Gu, F. R. Tay and P. Makvandi, Advances in antimicrobial microneedle patches for combating infections, Adv. mater., 2020, 32(33), 2002129.

20 M. Battisti, R. Vecchione, C. Casale, F. A. Pennacchio, V. Lettera, R. Jamaledin, M. Profeta, C. Di Natale, G. Imparato and F. Urciuolo, Non-invasive production of multi-compartmental biodegradable polymer microneedles for controlled intradermal drug release of labile molecules, Front. Bioeng. Biotechnol., 2019, 7, 296.

21 M. A. Badalamente and L. C. Hurst, Efficacy and safety of injectable mixed collagenase subtypes in the treatment of Dupuytren's contracture, J. Hand Surgery, 2007, 32(6), 767-774.

22 H. Alipour, A. Raz, S. Zakeri and N. D. Djadid, Therapeutic applications of collagenase (metalloproteases): a review, Asian Pac. J. Trop. Biomed., 2016, 6(11), 975-981.

23 Q. L. Wang, J. W. Ren, B. Z. Chen, X. Jin, C. Y. Zhang and X. D. Guo, Effect of humidity on mechanical properties of dissolving microneedles for transdermal drug delivery, J. Ind. Eng. Chem., 2018, 59, 251-258.

24 J. R. Elkinton, W. A. Wolff and W. E. Lee, Plasma transfusion in the treatment of the fluid shift in severe burns, Ann. Surg., 1940, 112(1), 150.

25 S. Y. Jo, C.-H. Lee, W.-J. Jung, S.-W. Kim and Y.-H. Hwang, Common features of atopic dermatitis with hypoproteinemia, Korean J. Pediatr., 2018, 61(11), 348-354.
26 A. McFarlane, Metabolism of plasma proteins, Mammalian protein metabolism, Elsevier, 1964, pp. 297-341.

27 G. T. Hermanson, Bioconjugate techniques, Academic Press, 2013.

28 S. Vaccaro, G. Gennari, L. Callegaro, A. Giannelli and S. Caruso, Fidia Farmaceutici SpA, Pharmaceutical compositions containing hyaluronic acid and collagenase for the topical treatment of wounds, burns and ulcers, US Pat., 8,900,576, 2014.

29 Y. Kim, S. O. Ho, N. R. Gassman, Y. Korlann, E. V. Landorf, F. R. Collart and S. Weiss, Efficient site-specific labeling of proteins via cysteines, Bioconjugate Chem., 2008, 19(3), 786-791.

30 K. Tanaka, N. Teramura, O. Hayashida, K. Iijima, T. Okitsu and S. Hattori, The C-terminal segment of collagenase in Grimontia hollisae binds collagen to enhance collagenolysis, FEBS Open Bio, 2018, 8(10), 1691-1702.

31 A. L. Silva, R. A. Rosalia, A. Sazak, M. G. Carstens, F. Ossendorp, J. Oostendorp and W. Jiskoot, Optimization of encapsulation of a synthetic long peptide in PLGA nanoparticles: low-burst release is crucial for efficient $\mathrm{CD}^{+} \mathrm{T}$ cell activation, Eur. J. Pharm. Biopharm., 2013, 83(3), 338-345.

32 M. Allahyari and E. Mohit, Peptide/protein vaccine delivery system based on PLGA particles, Hum. Vaccines Immunother., 2016, 12(3), 806-828.

33 S. Vaccaro, M. Caputo, C. Cuppari and G. Gennari, Fidia Farmaceuticals SpA, New process for the production and purification of the collagenase enzyme from vibrio alginolyticus, US Pat., 9,738,883, 2017.

34 G. Latour, L. Robinet, A. Dazzi, F. Portier, A. DenisetBesseau and M.-C. Schanne-Klein, Correlative nonlinear optical microscopy and infrared nanoscopy reveals collagen degradation in altered parchments, Sci. Rep., 2016, 6(1), 26344.

35 X. Chen, O. Nadiarynkh, S. Plotnikov and P. J. Campagnola, Second harmonic generation microscopy for quantitative analysis of collagen fibrillar structure, Nat. Protoc., 2012, $7(4), 654-669$.

36 Y. Hiraishi, T. Nakagawa, Y.-S. Quan, F. Kamiyama, S. Hirobe, N. Okada and S. Nakagawa, Performance and characteristics evaluation of a sodium hyaluronate-based microneedle patch for a transcutaneous drug delivery system, Int. J. Pharm., 2013, 441(1-2), 570-579.

37 M. A. Luzuriaga, D. R. Berry, J. C. Reagan, R. A. Smaldone and J. J. Gassensmith, Biodegradable 3D printed polymer microneedles for transdermal drug delivery, Lab Chip, 2018, 18(8), 1223-1230.

38 M. Zhou, A. R. Zheng and J. H. Yang, Superhydrophobic surfaces fabricated by replica molding and its applications, Wuli Huaxue Xuebao, 2007, 23, 1296-1300.

39 S. H. Park, T. W. Lim, D.-Y. Yang, H. J. Kong, R.-H. Kim, K.-S. Kim and K.-S. Lee, Fabrication of nano-precision PDMS replica using two-photon photopolymerization and vacuum pressure difference technique, Bull. Korean Chem. Soc., 2004, 25(8), 1119-1120.

40 P. Ronnander, L. Simon, H. Spilgies, A. Koch and S. Scherr, Dissolving polyvinylpyrrolidone-based microneedle systems 
for in vitro delivery of sumatriptan succinate, Eur. J. Pharm. Sci., 2018, 114, 84-92.

41 S. P. Sullivan, N. Murthy and M. R. Prausnitz, Minimally invasive protein delivery with rapidly dissolving polymer microneedles, Adv. Mater., 2008, 20(5), 933-938.

42 T. Pouyani and G. D. Prestwich, Functionalized derivatives of hyaluronic acid oligosaccharides: drug carriers and novel biomaterials, Bioconjugate Chem., 1994, 5(4), 339-347.

43 S. Fitzpatrick, J. F. McCabe, C. R. Petts and S. W. Booth, Effect of moisture on polyvinylpyrrolidone in accelerated stability testing, Int. J. Pharm., 2002, 246(1-2), 143-151.

44 P. Kapoor, S. Sachdeva and S. Sachdeva, Topical hyaluronic Acid in the management of oral ulcers, Indian J. Dermatol. Venereol., 2011, 56(3), 300-302.

45 G. Celetti, C. Di Natale, F. Causa, E. Battista and P. A. Netti, Functionalized poly(ethylene glycol) diacrylate microgels by microfluidics: in situ peptide encapsulation for in serum selective protein detection, Colloids Surf., B, 2016, 145, 21-29.

46 T. Fotticchia, R. Vecchione, P. L. Scognamiglio, D. Guarnieri, V. Calcagno, C. Di Natale, C. Attanasio, M. De Gregorio, C. Di Cicco and V. Quagliariello, Enhanced drug delivery into cell cytosol via glycoprotein H-derived peptide conjugated nanoemulsions, ACS Nano, 2017, 11(10), 9802-9813.

47 C. Di Natale, S. La Manna, A. M. Malfitano, S. Di Somma, D. Florio, P. L. Scognamiglio, E. Novellino, P. A. Netti and D. Marasco, Structural insights into amyloid structures of the C-terminal region of nucleophosmin 1 in type A mutation of acute myeloid leukemia, Biochim. Biophys. Acta, Proteins Proteomics, 2019, 1867(6), 637-644.

48 C. Di Natale, P. L. Scognamiglio, R. Cascella, C. Cecchi, A. Russo, M. Leone, A. Penco, A. Relini, L. Federici and A. Di Matteo, Nucleophosmin contains amyloidogenic regions that are able to form toxic aggregates under physiological conditions, FASEB J., 2015, 29(9), 3689-3701.

49 F. A. Mercurio, C. Di Natale, L. Pirone, R. Iannitti, D. Marasco, E. M. Pedone, R. Palumbo and M. Leone, The Sam-Sam interaction between Ship2 and the EphA2 receptor: design and analysis of peptide inhibitors, Sci. Rep., 2017, 7(1), 1-11.
50 F. A. Mercurio, C. Di Natale, L. Pirone, D. Marasco, E. Calce, M. Vincenzi, E. M. Pedone, S. De Luca and M. Leone, Design and analysis of EphA2-SAM peptide ligands: a multidisciplinary screening approach, Bioorg. Chem., 2019, 84, 434-443.

51 F. A. Mercurio, C. Di Natale, L. Pirone, M. Vincenzi, D. Marasco, S. De Luca, E. M. Pedone and M. Leone, Exploring the Ability of Cyclic Peptides to Target SAM Domains: A Computational and Experimental Study, ChemBioChem, 2020, 21(5), 702-711.

52 F. A. Mercurio, D. Marasco, C. Di Natale, L. Pirone, S. Costantini, E. M. Pedone and M. Leone, Targeting EphA2-Sam and Its Interactome: Design and Evaluation of Helical Peptides Enriched in Charged Residues, ChemBioChem, 2016, 17(22), 2179-2188.

53 F. A. Mercurio, L. Pirone, C. Di Natale, D. Marasco, E. M. Pedone and M. Leone, Sam domain-based stapled peptides: structural analysis and interaction studies with the Sam domains from the EphA2 receptor and the lipid phosphatase Ship2, Bioorg. Chem., 2018, 80, 602-610.

54 R. Jamaledin, R. Sartorius, C. Di Natale, R. Vecchione, P. De Berardinis and P. A. Netti, Recombinant Filamentous Bacteriophages Encapsulated in Biodegradable Polymeric Microparticles for Stimulation of Innate and Adaptive Immune Responses, Microorganisms, 2020, 8(5), 650.

55 M. Liu, J. Sun and Q. Chen, Influences of heating temperature on mechanical properties of polydimethylsiloxane, Sens. Actuators, A, 2009, 151(1), 42-45.

56 C. Di Natale, G. Celetti, P. L. Scognamiglio, C. Cosenza, E. Battista, F. Causa and P. A. Netti, Molecularly endowed hydrogel with an in silico-assisted screened peptide for highly sensitive small molecule harvesting, Chem. Commun., 2018, 54(72), 10088-10091.

57 F. Bassetto, N. Maschio, G. Abatangelo, B. Zavan, C. Scarpa and V. Vindigni, Collagenase from Vibrio alginolyticus cultures: experimental study and clinical perspectives, Surgical Innovation, 2016, 23(6), 557-562.

58 R. R. S. Thakur, I. A. Tekko, F. Al-Shammari, A. A. Ali, H. McCarthy and R. F. Donnelly, Rapidly dissolving polymeric microneedles for minimally invasive intraocular drug delivery, Drug Delivery Transl. Res., 2016, 6(6), 800-815. 